Sopel M, Stasyuk O, Kuznetsov V, Goncharova L, Hubskyi P. Regina computer system for intelligent monitoring, diagnostics, and management of railway power supply systems Diagnostyka 2021; 22(4): 77-88. https://doi.org/10.29354/diag/143744

\title{
REGINA COMPUTER SYSTEM FOR INTELLIGENT MONITORING, DIAGNOSTICS, AND MANAGEMENT OF RAILWAY POWER SUPPLY SYSTEMS
}

\section{Mykhailo SOPEL ${ }^{1}$, Oleksandr STASYUK ${ }^{2}$, Valeriy KUZNETSOV ${ }^{3}$, Lidiya GONCHAROVA ${ }^{4}$, Petro HUBSKYI ${ }^{5}$}

\author{
${ }^{1}$ Small Private Enterprise "ANIGER" Ukraine, Kyiv, Garmatna str., 2. E-mail: regina@ @regina.org.ua \\ ${ }^{2}$ Automation and Computer-Integrated Technologies of Transport department, State University Infrastructure and \\ Technologies, Kyiv, Ukraine, I. Ogienka, 19. st. email: Stas1946@lanet.kiev.ua \\ ${ }^{3}$ Electric Power Department, Railway Research Institute, Chlopickiego str., 50, Warsaw, Poland, e-mail: \\ vkuznetsov@ikolej.pl \\ ${ }^{4}$ Automation and Computer-Integrated Technologies of Transport department, State University Infrastructure and \\ Technologies, Kyiv, Ukraine, I. Ogienka, 19. st. email: ktarael@yandex.ru \\ ${ }^{5}$ Electric Power Department, Railway Research Institute, Chlopickiego str., 50, Warsaw, Poland, e-mail: \\ phubskyi@ikolej.pl
}

\begin{abstract}
The analysis of the problem of innovative transformation of power supply networks of railways made it possible to scientifically substantiate the direction of research related to an optimal strategy for computer monitoring and intellectualization of the processes of power supply of traction substations of railways. Conceptual approaches to the formation of a new model of intellectualization of power supply networks have been developed. Differential models of high organization of synchronous vector measurements are proposed, allowing to determine the comprehensive information content of the primary data. Based on the concept of smart energy, a set of differential models and methods of harmonic and correlation analysis of anomalous and transient processes occurring in power systems has been developed. The REGINA computer system has been designed and manufactured to carry out, in real-time, intelligent monitoring, diagnostics, identification of accidents, optimization of power consumption and expanding the range of market services in managing railway power supply networks. The REGINA system complies with the requirements of ISO 9001: 2015 and the German certification body DOS. Many actual results obtained during the operation of the REGINA on the railways are presented.
\end{abstract}

Key words: REGINA intelligent computer system, intelligent monitoring, diagnostics, control, power supply, railway, identification of accidents, optimization of power consumption, traction substation, synchronous vector measurements, comprehensive informational content.

\section{RELEVANCE}

Modern trends in scientific research in the development of the world electric power industry, the energy of railway transport, as well as research on the key directions of the evolution of innovative transformation of power supply systems have shown that the solution to the complex problem of optimizing power supply, creating energy-saving technologies and organizing high-speed failure-free transportation is possible by intellectualizing fast technological processes of power consumption $[1,2]$. The solution to the complex problem of the innovation and investment transformation of power supply systems for railways is closely related to the intellectualization of energy systems, based on the globally recognized SMART Grid technologies, the prospects of which are determined by the creation of a new model for the intellectualization of electrical networks, which reflects the integration of modern scientific, engineering and organizational solutions in the field computer, network and intelligent technologies [3]. The concept of intelligent energy is based on the principles of self-healing, selfregulation, a single information space, etc., which together represent a self-monitoring, analyzing, and reporting smart technology. The basis for the organization of an intelligent computer environment for optimal power supply management is an innovative model that reflects the results of interintegration of the topology of the electrical system and the architecture of an intelligent computer network capable of solving a set of tasks that traditionally belong to the class of creative ones [4]. 
Sopel M, Stasyuk O, Kuznetsov V, Goncharova L, Hubskyi P.: Regina computer system for...

The main thing in the formation of intelligent technologies based on SMART Grid is the organization of all-mode computer monitoring of the entire complex of parameters of the power system as the basis for full-scale information support. The use of modern technologies of distributed vector measurements WAMS (Wide Area Measurement System) in which, thanks to the use of functionally oriented microprocessor devices PMU (Phasor Measurement Unit) and the widespread use of GPS satellite navigation systems, have opened up enormous possibilities for carrying out, with high accuracy, synchronous measurements of mode parameters in various points and segments of the distributed power system topology [5, 6].

Modern systems of continuous computer monitoring of parameters displaying normal, abnormal, and transient modes allow direct observation of the dynamic characteristics of the power system. Direct measurement of frequency, amplitudes, phases of voltages and currents, as well as other parameters, at various points of the network, with a rigid reference to astronomical time and accuracy of several hundred nanoseconds, allows you to determine, in real-time, the coefficient of lowfrequency oscillations of the power system and the level of risk of instability. The primary data obtained in this way are used to study the processes occurring in the power system, to determine its properties and dynamic characteristics at a qualitatively new level, as well as to form new knowledge in the field of railway energy [7,8]. The use of more advanced intellectual means of computer analysis and visual presentation of processed and primary information opens up the possibility of identifying the sources of low-frequency oscillations, their types, features, forms, places of occurrence, and characteristics of abnormal modes, as well as the boundaries of current stability [9]. An opportunity opens up to solve the problem of registering complex types of disturbances and, due to this, to carry out a virtual decomposition of the power system into asynchronously operating parts, as well as to damp low-frequency oscillations that swing the system and lead to a decrease in power flows [10]. The technology of modern synchronous vector measurements contributed to the expansion of the concept of "smart energy", as the basis for the innovative transformation of traction power grids and the creation of intelligent power supply systems of a new generation, which are focused on the formation and accumulation of new knowledge in the field of railway energy to create modern energy-saving technologies, optimize power supply and improve quality functioning [11,12]. This fact is confirmed, first of all, by the fact that synchronous measurements of the entire set of parameters of the power system, recorded in the process of slippery computer monitoring, opened up the opportunity to significantly improve the information content of primary data displaying anomalous or transient modes [13].

The analysis of the latest scientific research carried out by domestic and foreign scientists showed that in scientific publications, not enough attention is paid to the issues of cybersecurity, creating mathematical models and methods of organizing computer systems for intelligent monitoring to study the entire dynamics of anomalous and transient processes occurring in power systems. In addition, insufficient attention has been paid to methods for determining the whole depth of information content of the registered, based on synchronous vector measurements, multidimensional primary information $[14,15]$.

\section{THE PURPOSE OF THE WORK}

Scientific substantiation of the problem and organization of innovative computer systems for allmode intelligent monitoring of electrical networks and electrical power equipment. Synthesis of mathematical models of increased intellectual complexity for determining the specific information content of stochastic primary data, reflecting the processes taking place in complex energy systems. Conducting a detailed computer analysis of the functioning of distributed energy objects, the external environment, and, based on the possibility of using the almost unlimited performance of distributed computing, creating cognitive models for imitating creative activity as the basis for the formation of intelligent technologies. Develop a computer system for intelligent monitoring of REGINA power grids, determining a set of performance indicators, characteristics, criteria, and dynamic features of power grids and power equipment in normal, abnormal, and emergency modes.

\section{INTELLIGENT MONITORING}

The conceptual basis for the creation of innovative computer monitoring systems is the need for a joint and coordinated solution of global problems of increased complexity and dimension, such as - analysis of the results of all-mode computer monitoring, including retrospective, technical 
Sopel M, Stasyuk O, Kuznetsov V, Goncharova L, Hubskyi P.: Regina computer system for...

diagnostics, and assessment of the state of electrical objects and systems, forecasting the reliability of their functioning and operational management of power consumption [16]. Despite the relative autonomy of these tasks, they are in systemic unity and interdependence one with one. Taking into account the wide variety of connections between events that appear in electric power systems, as well as the high speed of the main technological processes of power supply, for the formation of modern monitoring systems, we use promising directions associated with the integration of intelligent computer-oriented methods for modeling the above global problems. The latest achievements in the field of computer, smart, and telecommunication technologies, as well as the emergence of modern methods of automatic and operational control of power supply, to date, have made it possible to provide, in real time, control of the modes of operation of electric power facilities and systems, as well as quantitative and qualitative observation of rapidly changing mode parameters inherent in the features of electrical networks and railway facilities. For a complete analysis, it is enough to register the preliminary information before the appearance of an anomalous mode of the power supply system, as well as obtain anomalous data and information after the end of abnormal modes, in conjunction with auxiliary data registered synchronously in time and related to network protection. This fact stimulated the introduction of the concept of "slippery computer monitoring" [17].

Schematic implementation of an innovative computer monitoring system for railroad power supply networks and electrical power equipment is shown in Fig.1. Computer monitoring in the railway power industry is presented in the form of three levels - the level of traction substations, which mainly uses slippery computer monitoring of the parameters of the power grid modes; the level of the power supply distance where the computer monitoring of the secondary parameters of the traction power system modes is used; the third level of the railway on which intelligent computer monitoring is carried out. At the first level of traction substations of the power supply system, preliminary information is recorded using sensors that are located in different segments of the power supply network, power electrical equipment and relay, and microprocessor protection systems. The received primary data, in the form of analog, discrete and digital signals, are the basis for the formation of a multi-level information environment organized from a system-wide standpoint. Primary data are recorded using slippery computer monitoring and can be presented as follows [8,14]

$$
\begin{gathered}
i_{k}=F_{k}^{i}\left(i_{k}^{d}, i_{k}^{a}, i_{k}^{p}, U_{k}, t_{k}, D_{j}, \theta_{e}, f, \tau, U_{k}^{i}, Z_{k}^{i}, V_{k}^{i}\right) \\
u_{k}= \\
F_{k}^{u}\left(u_{k}^{d}, u_{k}^{a}, i_{k}^{p}, i_{k}, t_{k}, D_{j}, \theta_{e}, f, \tau, U_{k}^{u}, Z_{k}^{u}, V_{k}^{u}\right) \lambda_{k}= \\
F_{k}^{\lambda}\left(\lambda_{k}^{d}, \lambda_{k}^{a}, \lambda_{k}^{p}, i_{k}, u_{k}, t_{k}, \theta_{e}, f, \tau, U_{k}^{\lambda}, Z_{k}^{\lambda}, V_{k}^{\lambda}\right)
\end{gathered}
$$

$$
\begin{gathered}
D_{j}=F_{j}^{D}\left(D_{j}^{d}, D_{J}^{a}, D_{j}^{p}, i_{k}, u_{k}, t_{j}, \theta_{e}, f, \tau, U_{j}^{D}, V_{j}^{d}\right) . \\
\mathrm{k}=1,2 . . \mathrm{n}, \mathrm{j}=1,2 . . \mathrm{m},
\end{gathered}
$$

where $\lambda_{k}, \mathrm{Dj}$ - analog and discrete signals; $i_{k}^{d}, u_{k}^{d}, \lambda_{k}^{d}, D_{j}^{d}$ - pre-emergency mode signals $i_{k}^{a}, u_{k}^{a}, \lambda_{k}^{a}, D_{j}^{a} ; \quad$ - emergency mode signals; $i_{k}^{p}, u_{k}^{p}, \lambda_{k}^{p}, D_{j}^{p}$ - signals of the post-emergency mode; $f$ is the frequency; $t$ is the time interval of the pre-emergency mode; $\mathrm{U}_{\mathrm{k}}$ - parameters that determine synchronicity and sometimes registration; $\mathrm{Vk}$ parameters that characterize several features of the formation of primary information; $\theta$ - parameters of environmental data. The value $\theta_{e}$ and $t_{\mathrm{y}}$ can be written as

$$
\begin{gathered}
\theta_{e}=F_{e}^{\theta}\left(i_{k}, u_{k}, Z_{e}^{\theta}, t_{e}, U_{e}^{\theta}, V_{e}^{\theta}\right) \\
t_{\gamma}=F_{\gamma}^{t}\left(i_{k}, u_{k}, \theta_{e}^{t}, f, Z_{\gamma}^{t}, U_{\gamma}^{t}, V_{\gamma}^{t}\right), \gamma=1,2, . . \\
\quad \mathrm{z}, e=1,2, . . \mathrm{r.} .(2)
\end{gathered}
$$

We represent the primary data

$i_{k}, u_{k}, \lambda_{k}, D_{j}, t_{\gamma}, \theta_{e}$ in the form of sets

$G^{i}, G^{u}, G^{\lambda}, G^{D}, G^{t}, G^{\theta}$ or

$$
\left\{i_{k}\right\} \in G^{i} \neq \varnothing,\left\{u_{k}\right\} \in G^{u} \neq \varnothing, \quad\left\{\lambda_{k}\right\} \in
$$

$G^{\lambda} \neq \varnothing$

$\left\{D_{j}\right\} \in G^{D} \neq \varnothing, \quad\left\{t_{\gamma}\right\} \in G^{t} \neq \varnothing$,

$G^{\theta} \neq \varnothing$.

The procedure for the formation of a single information and time space can be represented as a set $\mathrm{G}$ as

$G=G^{i} \cup G^{u} \cup G^{\lambda} \cup G^{D} \cup G^{t} \cup G^{\theta} \neq \varnothing$

$G=G^{i} \cup G^{u} \cup G^{\lambda} \cup G^{D} \cup G^{t} \cup G^{\theta}=\varnothing$.

The process of informatization of operational and strategic decisions at the level of the power supply distance of the railway power grid is implemented using computer monitoring of the secondary parameters of the modes. Thanks to the calculation of a set of parameters, based on the primary information presented by expressions (1) - (4), quantitative and qualitative indicators of transient modes are determined, leading influences are formed to control energy flows and compensate for reactive energy, and also at the level of the power supply distance, optimization of power consumption, procedures selfhealing and self-diagnosis, and the like. Computer monitoring of the aggregate of quantitative and qualitative secondary parameters of the $S$ mode of fast-flowing technological processes of power supply for traction can be written in the form

$$
S=\Phi(i, u, \lambda, D, t, \theta, Z, U, V, \tau) .
$$


Sopel M, Stasyuk O, Kuznetsov V, Goncharova L, Hubskyi P.: Regina computer system for...

The main task in the process of computer monitoring at the level of the power supply distance is to optimize the operating modes of the power supply system in such a way that from an infinite number of operational and strategic control influences $Q^{i}=Q^{i}\left(Q_{1}^{i}, Q_{2}^{i}, \ldots Q_{n}^{i}\right)$ is found such $Q_{o p t}^{i}$ that it satisfies the system of constraints in the process the optimality criterion of the form



Fig. 1. Innovative system of all-mode computer monitoring of railway power supply networks

The determination, as a result of computer monitoring, of the secondary parameters of the functioning of the power grid opens up the opportunity to instantly respond to a variety of emergency situations and effectively ensure high technical and economic indicators of power supply. As a result of intelligent computer monitoring, realtime optimization of unbalanced power flows, quality and safety margin of the power system, energy-saving technologies is implemented and, most importantly, new knowledge is formed and accumulated in the process of functioning of the power supply system.

\section{ORGANIZATION OF SLIPPERY COMPUTER MONITORING}

In the process of slippery monitoring, it is supposed to register, in the memory of an intelligent computer environment, $\mathrm{n}$ values of the primary stochastic process of each parameter of the power system from the entire set for the time interval $\mathrm{T} 1$ as shown in Fig.2. Further, during the operation of the power system, the data of the $(n+1)$ th measurement of each parameter is written in place of the first value in the corresponding memory cell of the computer environment, the $(n+2)$ th - in the second cell, etc. mode, the registration process continues automatically. At the moment, based on appropriate criteria, the computer system will record the appearance of an abnormal or emergency mode, then the registered $n$ values of the primary stochastic processes of each of the entire set of parameters remain in memory and, in fact, represent the registered process of the pre-emergency mode of operation of the power supply system with duration T1. Next, the entire set of parameters with a duration of $\mathrm{T} 2$ is recorded, reflecting the emergency mode of the power system, and the data of all parameters of the power network are recorded, the post-emergency mode with a duration of $\mathrm{T} 3$. The registered information presented in the form of pre-emergency, emergency, and post-emergency modes are formed based on system-wide positions in the form of single information space for further use.

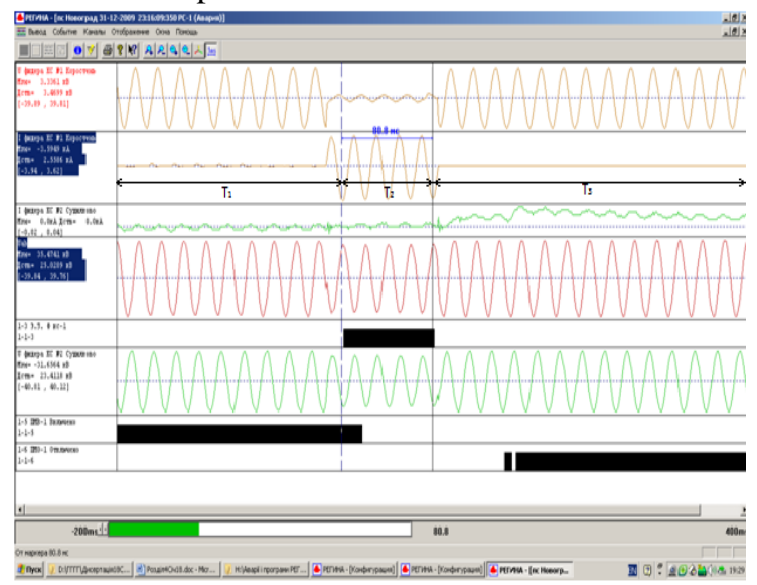

Fig. 2. Oscillograms of the organization of slippery monitoring of electrical railway networks

\section{DIFFERENTIAL MODELS OF SYNCHRONOUS VECTOR MEASUREMENTS}

Modern technologies of synchronous vector measurements are one of the most discussed areas of improving power supply networks. The problem of innovative transformation of power supply systems requires new organizational, scientific and engineering solutions in the field of information, intelligent and network technologies to optimize costs, improve the level of operational reliability, quality of electricity and expand the range of market opportunities [2,9].

The widespread use of the GPS satellite navigation system has opened up new opportunities to carry out synchronous measurements of a set of parameters at different points and segments of the 
Sopel M, Stasyuk O, Kuznetsov V, Goncharova L, Hubskyi P.: Regina computer system for...

power grid topology and register the values of angles, voltages and currents with a rigid reference to a single time. This fact opens up the opportunity to obtain a qualitatively new level of knowledge about the properties of the power supply system and the processes that take place in it $[1,6]$.

Each of the entire set of primary parameters representing the instantaneous values of currents and voltages of the direct reverse and zero sequences, including their harmonic components, as well as the values of phase angles in different nodes of the power grid, the rate of their change, asymmetry coefficients and non-sinusoidal voltage, the topology of the power grid synchronously recorded at various points $\left\{x_{j}\left(t_{i}\right)\right\} \in G_{j}^{t_{i}} \neq \varnothing$ is denoted in this case, we will assume that for each $\mathrm{jth}$ parameter of $x_{j}\left(t_{i}\right)$ the power grid $\left({ }^{j t h}=1,2, . . m\right)$, at the selected times $t_{i}$ $(i=1,2, \ldots n)$, synchronous registration is sequentially implemented $i^{\text {th }}$ instantaneous value. Then, the set of mode parameters can be represented as a vector of the form $X_{j}^{i}=\left(x_{j}\left(t_{i}\right), \tau_{j}^{i}, z_{\mathrm{r}}\right)^{t}, \quad(i=$ $1,2, . . n),(\mathrm{j}=1,2, . . \mathrm{m}),(\mathrm{r}=1,2, . . \mathrm{s})$, where $x_{j}\left(t_{i}\right)$ is the instantaneous value of the $j$ th parameter; $\tau_{j}^{i}$ - a value that characterizes the synchronism of measurements of parameters $x_{j}\left(t_{i}\right)$ in different nodes of the power supply network; $z_{r}$ is the value of the rth segment of the power supply network topology. Such a presentation of synchronous measurements of a set of primary parameters opens up the possibility of forming preliminary information in the form of a single information space from system-wide positions in the form

$\left\{X_{j}^{i}\right\} \in G_{j}^{i} \neq \varnothing, G^{\Theta}=\cup_{j=1}^{m} G_{j}^{i} \neq \varnothing$.

$X_{j}^{i}=\left(x_{j}\left(t_{i}\right), \tau_{j}^{i}, z_{\mathrm{r}}\right)^{t} \ldots \quad(i=1,2, . . n),(\mathrm{j}=1,2, . . \mathrm{m})$, $(\mathrm{r}=1,2, . . \mathrm{s})$

The obtained primary data, which are registered based on synchronous vector measurements and formed in a single information space, are used to synthesize mathematical models oriented to determine their full information content.

To synthesize such mathematical models, which open up the possibility of significantly expanding the technologies of distributed synchronized vector measurements in the power industry, we use modern methods of differential transformations Pukhovaya [18] represented by the following pair of mathematical expressions in the form

$$
\begin{gathered}
X_{j}^{i}(k)=\frac{H^{k}}{k !}\left[\frac{d^{k} x_{j}\left(t_{i}\right)}{d t^{k}}\right]_{t_{i}} \doteq x_{j}\left(t_{i}\right)= \\
\sum_{k=0}^{k=\infty}\left(\frac{t}{H}\right)^{k} X_{j}^{i}(k),
\end{gathered}
$$

where $x_{j}\left(t_{i}\right)$ is the primitive function of the argument $t$ which can be differentiated ntimes and which has many corresponding restrictions, including its derivatives; $X_{j}^{i}(k)$ - - differential Timage of the primeval function $x_{j}\left(t_{i}\right) ;-H$ a scale factor, the dimension of which coincides with the size of the argument $t$, as a rule, is selected on the basis $0 \leq t \leq H$ of the entire range of the function to the original $x_{j}\left(t_{i}\right) ; \quad \mp \quad-$ the symbol of the correspondence between the function - the original $x_{j}\left(t_{i}\right)$ and its differential T - image $X_{j}^{i}(k)$.

Based on direct differential transformation, which is located to the left of the symbol $\overline{-}$, a differential Timage of the function is formed $-x_{j}\left(t_{i}\right)$ to the original in the form of a discrete function $X_{j}^{i}(k)$ of an integer argument $\mathrm{k}=0,1,2, \ldots$ Based on the set of values of the $\mathrm{T}$-discrete function of the integer argument $X_{j}^{i}(k), \mathrm{k}=0,1,2, . .$, using the inverse differential transformation located to the right of the symbol $\bar{\perp}$, we obtain the original $x_{j}\left(t_{i}\right)$ function. Note that for $\mathrm{k}=0$, according to (8), for any instantaneous value $t_{i}$ of each jth parameter $x_{j}\left(t_{i}\right)$, the equality $x_{j}\left(t_{i}\right)=X_{j}^{i}(0)$ holds. Due to this, on the basis $x_{j}\left(t_{i}\right)=\sum_{k=0}^{k=\infty}\left(\frac{t}{H}\right)^{k} X_{j}^{i}(k)$,of the form we form a system of algebraic equations of the $\mathrm{n}$-th order, which can be written in the form

$\frac{t_{1}}{H} X_{j}^{i}(1)+\left(\frac{t_{1}}{H}\right)^{2} X_{j}^{i}(2)+\left(\frac{t_{1}}{H}\right)^{3} X_{j}^{i}(3)+. .+\left(\frac{t_{1}}{H}\right)^{n} X_{j}^{i}(n)$

$$
=x_{j}\left(t_{i}\right)-X_{j}^{i}(0)
$$

$\frac{t_{2}}{H} X_{j}^{i}(1)+\left(\frac{t_{2}}{H}\right)^{2} X_{j}^{i}(2)+\left(\frac{t_{2}}{H}\right)^{3} X_{j}^{i}(3)+. .+\left(\frac{t_{2}}{H}\right)^{n} X_{j}^{i}(n)=$

$x_{j}\left(t_{i+1}\right)-X_{j}^{i}(0)$

$\frac{t_{n}}{H} X_{j}^{i}(1)+\left(\frac{t_{n}}{H}\right)^{2} X_{j}^{i}(2)+\left(\frac{t_{n}}{H}\right)^{3} X_{j}^{i}(3)+. .+\left(\frac{t_{n}}{H}\right)^{n} X_{j}^{i}(n)$

$$
=x_{j}\left(t_{n}\right)-X_{j}^{i}(0) \text {. }
$$

$(i=1,2, . . n), \quad(\mathrm{j}=1,2, . . \mathrm{m})$

As an example, consider the graph of the anomalous power system mode shown in Fig.3.a. An abnormal or emergency process of supplying electricity is represented by a set of instantaneous values of current

$i\left(t_{0}\right), i\left(t_{1}\right), i\left(t_{2}\right), \ldots i\left(t_{j}\right), \ldots i\left(t_{m}\right), \quad$ or, respectively, the voltage $u\left(t_{0}\right), u\left(t_{1}\right), u\left(t_{2}\right), . u\left(t_{j}\right), \ldots u\left(t_{m}\right)$, over the entire interval of the non-stationary process 
Sopel M, Stasyuk O, Kuznetsov V, Goncharova L, Hubskyi P.: Regina computer system for...



Fig. 3. Abnormal mode a, the differential image of anomalous mode $b$.

Differential image of the anomalous mode of instantaneous current values $i\left(t_{0}\right), i\left(t_{1}\right), i\left(t_{2}\right), \ldots i\left(t_{j}\right), \ldots i\left(t_{m}\right)$, after performing a set of transformations (8), (9)

Based on direct differential transformation $I_{0}(k)=\frac{H^{k}}{K !}\left[\frac{d^{k} i(t)}{d t^{k}}\right]_{t=t_{0}}$, according to expression (8), the following equalities take place $i\left(t_{0}\right)=I_{0}(0)$, $i\left(t_{1}\right)=I_{1}(0), . . \quad . . \quad i\left(t_{j}\right)=I_{j}(0), \quad \mathrm{i}\left(\mathrm{t}_{\mathrm{m}}\right)=\mathrm{I}_{\mathrm{m}}(0)$, using which and uncoupling the system of algebraic equations (9) for the case $j=0$, we obtain the next set of T-discrete functions of an integer argument, which are an image $i\left(t_{0}\right)$ at a point $\left(t_{0}\right)$, as shown in fig.3.b

Based on the analysis of the results obtained $I_{0}(0), I_{0}(1), I_{0}(2), I_{0}(3), I_{0}(4)$, one can draw attention to the fact that the function $i(t)$ is represented at a point (Fig.3.b) is not only the magnitude of its instantaneous value $i\left(t_{0}\right)=I_{0}(0)$, , but also a set of $I_{0}(1), I_{0}(2), \ldots I_{0}(k)$ T-discrete , each $k$ of which $k$ is equivalent - the derivative of $i(t)$ the functionat the same point $t_{0}$. Thus, the complete information content of the registered primary data is determined by the fact that when processing them under (8), (9), not only instantaneous values $i\left(t_{0}\right)=I_{0}(0)$, of parameters at points are used $t_{j}$, but also the $n$ values of derivatives points.

Such a procedure for intellectualizing the processes of determining the informativeness of the parameters of the modes of intelligent power supply networks of railways makes it possible to eliminate the deficit of registered primary information, which reflects the fast technological processes of power supply and is the basis for the formation and accumulation of new knowledge in the field of optimization and energy saving of railway power systems.

In the general case, having solved the system of equations (9), we obtain, for all instantaneous values of $t_{i}$ each jth parameter $x_{j}\left(t_{i}\right)$, a set of $x_{j}\left(t_{i}\right)=$ $X_{j}^{i}(0), X_{j}^{i}(1), X_{j}^{i}(2), X_{j}^{i}(3)$, T-discrete $\ldots X_{j}^{i}(n)$ After analyzing the results obtained $\quad X_{j}^{i}(k),(i=$ $1,2, . . n), \quad(j=1,2, . . m)$, one can draw attention to the fact that as a result of the calculations performed according to (8), (9), the vector $X_{j}^{i}=$ $\left(x_{j}\left(t_{i}\right), \tau_{j}^{i}, z_{\mathrm{r}}\right)^{t}, \quad$ given $X_{j}^{i}(0)=x_{j}\left(t_{i}\right), \quad$ can be represented as follows

$X_{p j}^{i}=\left(X_{j}^{i}(0), X_{j}^{i}(1), X_{j}^{i}(2), \ldots X_{j}^{i}(n), \tau_{j}^{i}, z_{\mathrm{r}}\right)^{t},(10)$

$(i=1,2, . . n), \ldots \quad(\mathrm{j}=1,2, . . \mathrm{m}),(\mathrm{r}=1,2, . . \mathrm{s})$

which became possible thanks to the formed mathematical models (7) - (10). With this approach, the valueof the jth parameter $x_{j}\left(t_{i}\right)$ at each I (i) ${ }^{\text {th }}$ point $X_{j}^{i}=\left(x_{j}\left(t_{i}\right), \tau_{j}^{i}, z_{\mathrm{r}}\right)^{t}$ is represented not only by its instantaneous value at the same point but also additionally by a set of T-discrete $X_{j}^{i}(1)$, $X_{j}^{i}(2), \ldots X_{j}^{i}(n)$ of which $(\mathrm{k}=1,2, . . n)$ is equivalent to the kth derivative of the jth parameter $x_{j}\left(t_{i}\right)$ at the point $t_{i}$.Thus, the full informativeness of the registered primary $\operatorname{data} X_{p j}^{i}=\left(X_{j}^{i}(0), X_{j}^{i}(1)\right.$, $\left.X_{j}^{i}(2), \ldots X_{j}^{i}(n), \tau_{j}^{i}, z_{\mathrm{r}}\right)^{t}$, is determined due to the fact that during their processing, not only the instantaneous values of the parameters at the points are used $t_{i}$, but also the value $n$ of the derivatives at the same points.

This approach makes it possible to eliminate the lack of information on fast-flowing electromechanical transients, which are very important when conducting research and adequate analysis of the dynamic characteristics of power supply networks and determining their state, power fluctuations, generator loads, emergency locations and a combination of other parameters.

\section{DIFFERENTIAL MINING MODELS}

Based on the obtained results of synchronous vector measurements of a set of stochastic processes reflecting the normal or abnormal regime of the power system, presented in the form of vectors $X_{p j}^{i}=$ $\left(X_{j}^{i}(0), X_{j}^{i}(1), X_{j}^{i}(2), \ldots X_{j}^{i}(n), \tau_{j}^{i}, z_{\mathrm{r}}\right)^{t}$, we will form 
Sopel M, Stasyuk O, Kuznetsov V, Goncharova L, Hubskyi P.: Regina computer system for...

many differential mathematical models of increased intellectual complexity and dimension, focused on gaining new knowledge in the process of intelligent analysis of transient fast processes.

Studies of anomalous and emergency modes occurring in the power supply systems of railways have shown that interference caused by individual harmonic components of the emergency process has a great influence on the reliability of the computer systems for power supply control and railway automation. In this regard, differential images of the registered primary data of current values $i(t)$, based on the implementation of procedures (8), (9), are presented in the form $I_{j}(0), I_{j}(1), \ldots I_{j}(k), \ldots I_{j}(n)$, and are used to calculate individual harmonic components of the emergency mode at each $j^{\check{u}}$ point of the emergency process.

The direct Fourier transform on a finite interval $(0, \mathrm{~T})$ is represented as

$\dot{I}_{\gamma}=\frac{j^{2}}{T} \int_{0}^{T} e^{-j \gamma \omega t} i(t) d t$

where is $\dot{I}_{\gamma}$ the complex amplitude $\gamma^{\breve{u}}$ of the current harmonic; $\omega=\frac{2 \pi}{T}$ - frequency of the fundamental harmonic; $j^{2}=-1$.

Let us use, under (8), the inverse differential transformation $i\left(t_{j}\right)=\sum_{k=0}^{k=\infty}\left(\frac{t}{H_{j}}\right)^{k} I_{j}(k)$ and write

$$
\dot{I}_{\gamma}=\sum_{k=0}^{k=\infty} \frac{I_{j}(k)}{H_{j}^{k}}\left(\frac{j^{2}}{T} \int_{0}^{T} t^{k} e^{-j \gamma \omega t} d t\right) .
$$

The value of the integral in expression (12) can be represented by the following relationship $[11] \int_{0}^{T} t^{k} e^{-j \gamma \omega t} d t=\frac{k !(-1)^{k}}{(-j \gamma \omega t)^{k+1}}(1-$ $\left.\sum_{m=0}^{m=k} \frac{(-j \gamma \omega T)^{m}}{m !}\right)$.

By substituting expression (13) into (12) and performing the appropriate transformations, we obtain a mathematical model for determining the required set of complex $\dot{I}_{\gamma} \gamma^{x}$ harmonic amplitudes

$\dot{I}_{\gamma}=\frac{1}{\pi \gamma} \sum_{k=0}^{k=\infty} \frac{k !}{\left(j \gamma \omega H_{l}\right)^{k}}\left[\sum_{m=0}^{m=k}\left(\frac{T}{H_{j}}\right)^{m} \frac{(-j \gamma \omega t)^{m}}{m !}-\right.$

$1] I_{l}(k)$.

The mathematical model (14) is fundamental in the study of abnormal modes. Thanks to its application, it becomes possible to use the full awareness of the primary data presented in the $\mathrm{T}$ region by the expression to form new knowledge about abnormal modes of power supply systems of railways.

In those cases when, in the process of studying the spectral characteristics of anomalous modes of power systems, it is necessary to apply the Fourier transform with infinite limits and restrictions $i(t)=0$ at $t<0$ , which can be written as $[6,13]$

$I(j \omega)=\int_{0}^{\infty} e^{-j \omega t} i(t) d t$,

$i(t)=$

$\frac{1}{2 \pi j} \int_{-j \infty}^{j \infty} e^{j \omega t} I(j \omega) d t \omega,(15)$

then, having implemented the appropriate mathematical transformations, similar to the above, we synthesize a mathematical model for determining the spectral density by processing the primary information $I_{j}(0), I_{j}(1), \ldots I_{j}(k), \ldots I_{j}(n)$ presented in the T-region

$I(j \omega)=\sum_{k=0}^{k=\infty} \frac{k !}{\left(j \omega H_{l}\right)^{k}} \frac{I_{l}(k)}{j \omega}$,
$\frac{1}{2 \pi j} \int_{-j \infty}^{j \infty} \frac{\left(j \omega H_{l}\right)^{k}}{k !} I(j \omega) d j \omega$.

$I_{j}(k)=$

The mathematical expression (16) allows calculating the value of the spectral density of the emergency mode, using for this preliminary information $I_{j}(0), I_{j}(1), \ldots I_{j}(k), \ldots I_{j}(n), \quad$ which displays in the T-region anomalous processes occurring in the power system for in-depth research and the formation of new knowledge.

Suppose the issues of using the primary information $I_{j}(0), I_{j}(1), \ldots I_{j}(k), \ldots I_{j}(n)$ presented in the T-region by an expression for carrying out the correlation analysis of anomalous processes. The calculation of the cross-correlation function $R\left(i_{s}\left(t_{l}\right) i_{f}\left(t_{l+r}\right)\right)$ of two variables $i_{s}\left(t_{l}\right), i_{f}\left(t_{l+r}\right)$ can be written in the form $R\left(i_{s}\left(t_{\xi}\right) i_{f}\left(t_{3+r}\right)\right)=$ $\frac{1}{N} \sum_{e=1}^{N} i_{s}\left(t_{\xi}\right) i_{f}\left(t_{\xi+r}\right)$. (17)

$s=1,2, \ldots n, \quad f=1,2, \ldots N, \quad \xi=1,2, \ldots P$, $r=1,2, \ldots m$

Using differential transformations (1), we write the expression (14) in the image area as

$R_{S f}(k)=\frac{1}{N} \sum_{\xi=1}^{N} \sum_{e=0}^{e=k} I_{S}^{\xi}(l) I_{f}^{\xi+r}(k-l)$

and, using the differential representation of the primary information $I_{j}(0), I_{j}(1), \ldots I_{j}(k), \ldots I_{j}(n)$, we organize the computational process in accordance with (18) as follows. When $k=0$

$R_{S f}(0)=\frac{1}{N} \sum_{\xi=1}^{N} I_{S}^{\xi}(0) I_{f}^{\xi+r}(0)$.

Accordingly, for, $k=1$ we obtain a $R_{S f}(1)$ discrete as follows

$R_{S f}(1)=\frac{1}{N} \sum_{3=1}^{N}\left(I_{S}^{3}(0) I_{f}^{3+r}(1)+I_{S}^{3}(1) I_{f}^{3+r}(0)\right)$

Similarly, for $k=2$, we get $R_{S f}(2) R_{S f}(2)=$ $\frac{1}{N} \sum_{\xi=1}^{N}\left(I_{S}^{\xi}(0) I_{f}^{\xi+r}(2)+I_{S}^{\xi}(1) I_{f}^{\xi+r}(1)+\right.$

$\left.I_{S}^{\xi}(2) I_{f}^{\xi+r}(0)\right)$. 
Sopel M, Stasyuk O, Kuznetsov V, Goncharova L, Hubskyi P.: Regina computer system for...

After calculating the corresponding number of $R_{S f}(k)$ discrete $k=0,1,2, .$. and applying the inverse differential transformation (8), we obtain $R\left(i_{s}\left(t_{\xi}\right) i_{f}\left(t_{\xi+r}\right)\right)$ in the following form

$$
R\left(i_{s}\left(t_{\xi}\right) i_{f}\left(t_{\xi+r}\right)\right)=R_{S f}(0)+R_{S f}(1) \frac{t}{H}+
$$

$R_{S f}(2) \frac{t^{2}}{H^{2}}+.$.

Applying the well-known provisions of the theory of differential transformations to represent the registered primary information in the image area $j^{\check{u}}$ at each point, in the form of a set of discrete samples $\quad I_{j}(0), I_{j}(1), \ldots I_{j}(k), \ldots I_{j}(n)$ of the investigated emergency process, it is possible to significantly expand modern technologies of synchronous vector measurements, in terms of determining the entire depth of information content of the obtained primary data. In addition, based on the presentation of preliminary information in the field of images, it becomes possible to form new computeroriented methods for analyzing the modes of operation of power systems aimed at the formation and accumulation of new knowledge in this area.

\section{REGINA INTELLIGENT MONITORING SYSTEM}

As a result of the research carried out by the private enterprise "ANIGER" and the IED of the National Academy of Sciences of Ukraine, the conceptual foundations of reasonable railway energy have been formed, which reflects the intelligent interaction of pricing, technological processes of power supply and the efficiency of resource use. Methods of computer intellectualization of power supply processes and an information model providing deep mutual integration of power grid and computer infrastructure have been investigated. Differential methods of harmonic analysis of anomalous modes of power systems for the formation of energy-saving control procedures have been developed. Models of identification of emergency modes are synthesized. Differential mathematical models of cybersecurity of a computer environment for dynamic power supply control have been investigated, which opened up the possibility of optimizing network planning, regulating loads, conducting continuous monitoring to account for and analyze the occurrence and development of technological disruptions. A methodology has been developed for organizing the computer infrastructure for managing the energy supply system of railways, which covers all subjects of the electricity market.
Based on the research results, the REGINA computer system has been developed, which is designed to carry out, in real time, intelligent monitoring, diagnostics, optimization, energy-saving, and expanding the range of market services in the process of managing railroad power supply networks. The REGINA system is made in the form of a set of modules, a variant of the typical architecture shown in Fig.4 in the form of a local computer network.

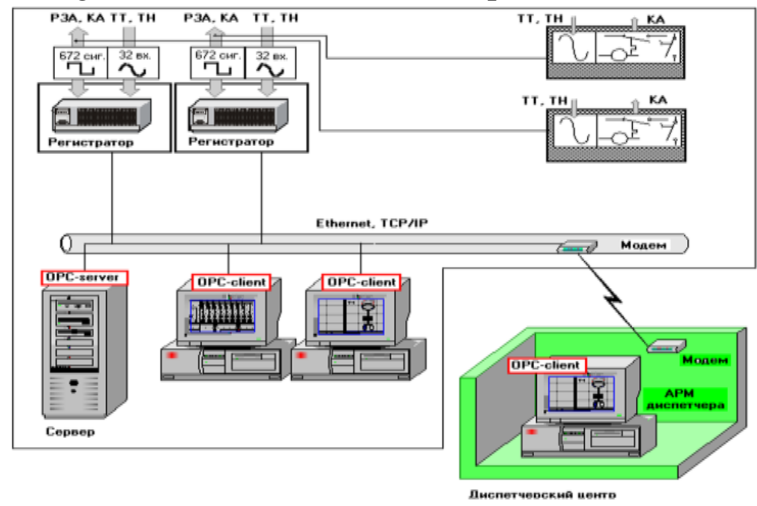

Fig. 4. A variant of a typical module of the REGINA architecture presented in the form of a local computer network

Brief technical characteristics of the REGINA system module:

- number of input analog signals - 32;

- number of input discrete signals - 224;

- $\quad$ sampling frequency $-1000 \div 2000 \mathrm{~Hz}$;

- duration of registration of one emergency event - not less than $12 \mathrm{~s}$;

- duration of registration of the pre-emergency mode $-0.1 \div 0.9 \mathrm{~s}$;

- the transition to the registration of the emergency mode is carried out when:

- going beyond the analog signal settings,

- change in the state of discrete signals;

- current variation range - from 0.01 to $300 \mathrm{~A}$;

- $\quad$ voltage range - from 0.01 to $400 \mathrm{~V}$;

- registration of discrete signals of the "dry contact" type and potential;

- synchronous registration of analog and discrete signals.

REGINA computer infrastructure for managing the power supply system at the level of the railway traction substation (Fig.5) covering all subjects of the electricity market provides intelligent monitoring, diagnostics and forecast of operating modes, optimization of energy consumption, implementation of energy saving procedures, and expansion of the range of market services.

The main tasks performed by the REGINA system include: registration of analog and discrete signals, analysis of the development of emergencies, assessment of the functioning of relay protection and 
Sopel M, Stasyuk O, Kuznetsov V, Goncharova L, Hubskyi P.: Regina computer system for...



Fig. 5. Computer infrastructure of REGINA on traction substation automation devices, determination of fault locations in case of short circuits on feeders of the railroad overhead contact network, DPR, signaling system, calculation of the residual life of high-voltage circuit breakers, phase and harmonic analysis of sinusoidal signals, separation of symmetrical components in three-phase networks of alternating voltage, as well as carrying out, in real-time, total dynamic control of the entire set of parameters.

Here are many indicators in firures, characteristics, and various abnormal operating modes of the railway power supply system, which are registered and processed by the REGINA intelligent monitoring system.

a)



b)

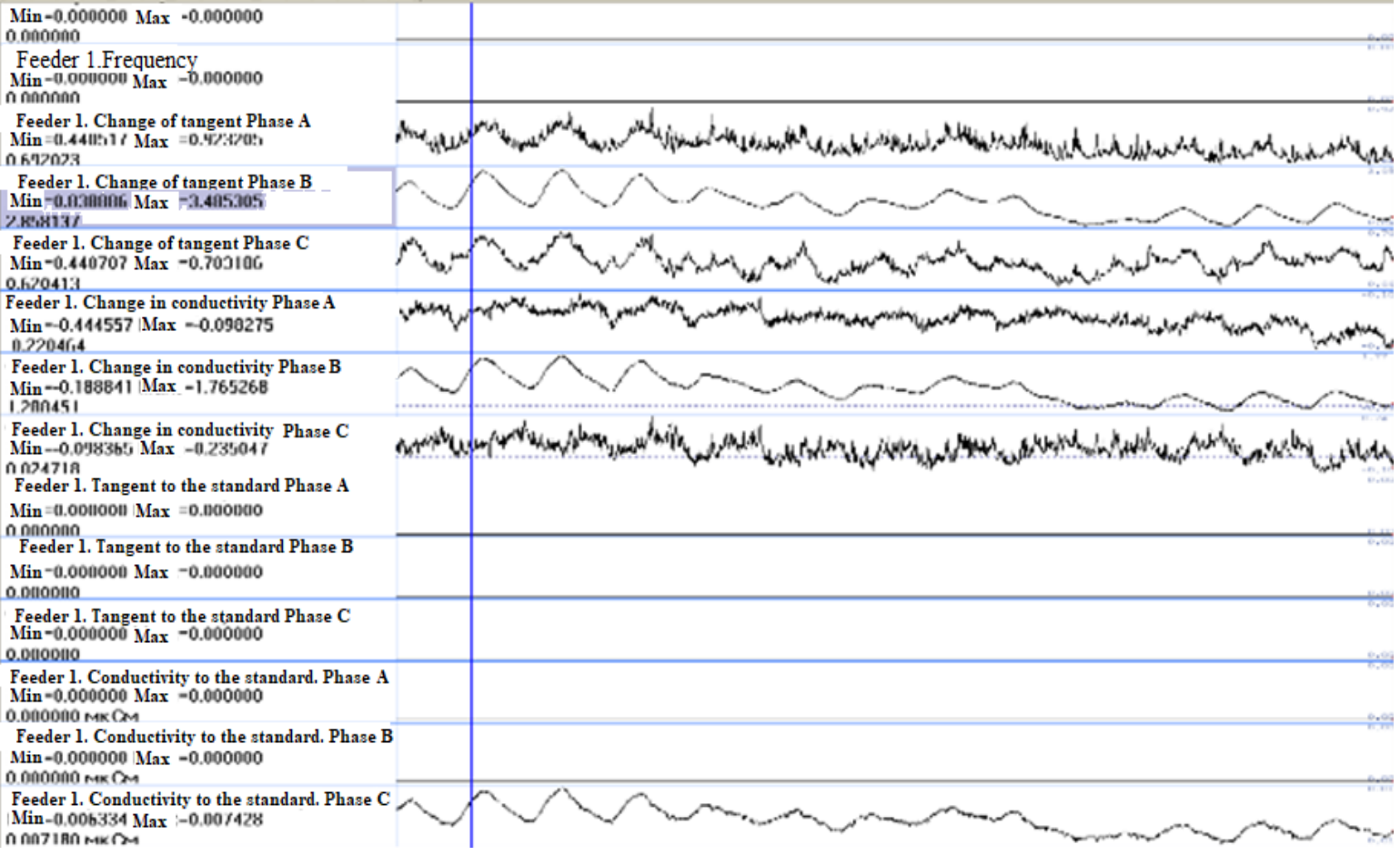

Fig. 6. Intelligent monitoring and the results of determining the insulation parameters of high-voltage bushings of power transformers during operation: a) power transformer; b) a set of parameters determined by the REGINA system 
Sopel M, Stasyuk O, Kuznetsov V, Goncharova L, Hubskyi P.: Regina computer system for...

a)



b)
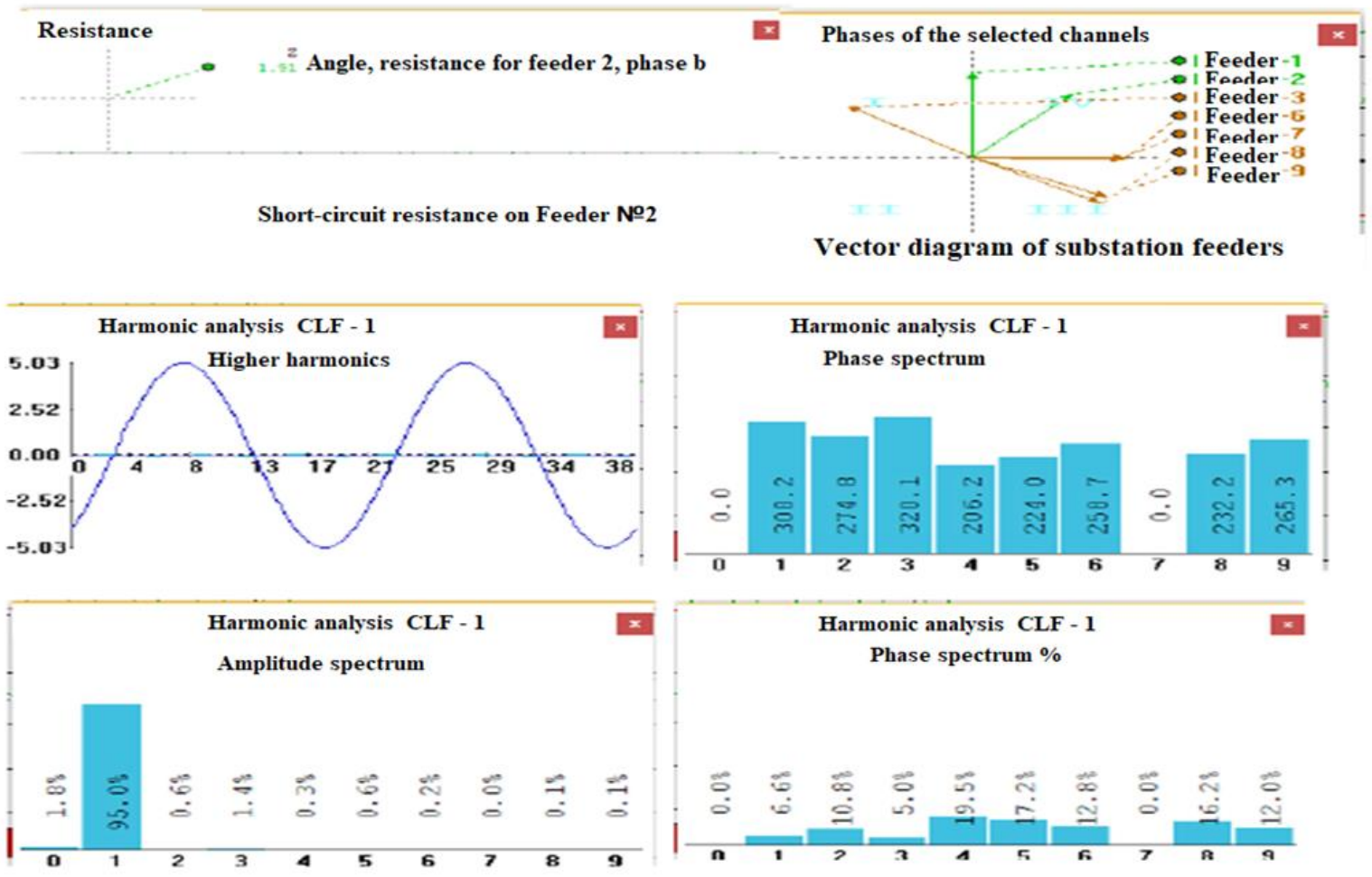

Fig. 7. Examples of emergency information generated by the REGINA system for making operational and strategic decisions: a) received from relay protection devices; b) obtained from the railway AC power supply system. 
Sopel M, Stasyuk O, Kuznetsov V, Goncharova L, Hubskyi P.: Regina computer system for...

The company - the manufacturer of the REGINA computer system for intelligent monitoring, diagnostics, and management of power supply networks of railways, certified for compliance with the requirements of international standards for the quality management system ISO 9001: 2015, received a certificate from the German certification body DOS, as well as from the National Accreditation Agency of Ukraine.

\section{CONCLUSION}

1. Analysis of the innovative transformation of power supply networks of railways and their evolution allowed to scientifically substantiate scientific research related to the creation of intelligent computer networks and intelligent power supply systems based on globally recognized SMART Grid technologies. Conceptual approaches have been developed to form a new model of intellectualization of power supply networks, the dominant feature of which is the formation and accumulation of new knowledge in the relevant subject area.

2. Based on fundamental and applied research in the field of mathematical and computer modeling, methods of organizing intelligent computer systems for managing complex energy objects, processes, and phenomena are substantiated, conceptually based on the use of the necessary or excess computing performance at any point or segment of the object's topology.

3. Differential mathematical models of high intellectual complexity and dimensions are proposed for organizing synchronous vector measurements, which allow determining the comprehensive information content of the primary data obtained by synchronous registration of the entire set of parameters of the modes of energy objects and systems.

4. Based on the concept of smart energy, a set of differential mathematical models and methods of harmonic and correlation analysis of anomalous and transient processes occurring in power systems, determination of individual harmonic components of stochastic processes of their features has been developed. This opens up additional opportunities for solving the problem of innovative transformation of power supply networks.

Based on the research results, the REGINA computer system has been developed, which is designed to carry out, in real time, intelligent monitoring, diagnostics, optimization, energy saving, and expanding the range of market services in the process of managing railroad power supply networks. The REGINA system complied with the requirements of international quality management system standards ISO 9001: 2015 and received a certificate from the German certification body DOS. REGINA has been put into operation at more than two hundred traction substations. Many actual results obtained during the process of the REGINA are carried out, in particular, graphs of intelligent analysis of emergency modes of DC and AC power supply systems. Results of identification of emergency mode obtained based on thoughtful harmonic analysis. The results of monitoring the abnormal mode of synchronization with the operation of the protection system and the operation of high-voltage circuit breakers are presented. The graphs for intelligent monitoring of traction transformers and intelligent analysis of abnormal and emergency modes of the railway power supply system are presented.

\section{REFERENCE}

1. Kulbovskyi I, Holub HM, KyiashkoVT, Andonova S. Information model of railway transport power supply system computer monitoring data flow. Metallurgical and Mining Industry. 2018; 2: 31-36.

2. Sopel MF, Grebchenko MV, Maksimchuk VF, Pilipenko Yu.V. Determining the location of a singlephase earth fault in the conditions of electromagnetic influence on overhead signaling, centralization and blocking lines of railways. Technical electrodynamics. 2019;1:50-54.

https://doi.org/10.15407/techned2019.01.050.

3. Yoldaş Y. Önena A, Muyeen SM. Vasilakos AV. Alan I. Enhancing smart grid with microgrids: Challenges and opportunities. Renewable Sustainable Energy Rev. 2017;72:205-214. https://doi.org/10.1016/j.rser.2017.01.064

4. Stasiuk A. Kuznetsov V, Goncharova L., Hubskyi P. Models of the computer intellectualization optimal strategy of the power supply fast-flowing technological processes of the railways traction substations. Komunikácie. 2021; 23(2):30-C36. https://doi.org/10.26552/com.C.2021.2.C30-C36

5. Bartłomiejczyk M. Smart grid technologies in electric power supply systems of public transport. Transport. 2018;33(5):1144-1154

https://doi.org/10.3846/transport.2018.6433 .

6. Brenna M, Foiadelli F, Kaleybar HJ. The evolution of railway power supply systems toward smart microgrids: The concept of the energy hub and integration of distributed energy resources. IEEE Electrification Magazine. 2020;8(1):12-23 https://doi.org/10.1109/MELE.2019.2962886.

7. Zhelieznov KI, Akulov AS, Zabolotnyi OM, Ursulyak LV, Chabanuk EV, Shvets AO, Kuznetsov VG, Radkevych AV The revised method for calculating of the optimal train control mode. Archives of Transport. 2019;51(3):21-34.

https://doi.org/10.5604/01.3001.0013.6160

8. Holub H, Kulbovskyi I, Skliarenko I, Hannoshyna I, Klochkov Y, Kharuta V. Research on the possibilities of solution of the monitoring projects of the railway power supply system. Technol. Aud. Prod. Reserv. Private Company Technology Center, 2019;6(50): $23-$ 25. https://doi.org/10.15587/2312-8372.2019.187278

9. Kaleybar HJ, Brenna M, Foiadelli F, Fazel SS, Zaninelli D. Power quality phenomena in electric railway power supply systems: an exhaustive framework and classification. Energies. 2020; 13(24):6662. https://doi.org/10.3390/en13246662 
Sopel M, Stasyuk O, Kuznetsov V, Goncharova L, Hubskyi P.: Regina computer system for...

10. Gomez-Exposito A, Conejo AJ, Canizares C. Electric energy systems: analysis and operation. CRC Press. 2018.

11. Stasiuk AI, Goncharova LL. Mathematical models of computer intellectualization of technologies for synchronous phasor measurements of parameters of electric networks. New Means Cybernetics, Informatics, Computers Engineering And Systems Analysis. Springer Science. 2016;52(5):825-830. https://doi.org/10.1007/s10559-016-9883-5.

12. Szeląg A. Trakcja elektryczna - podstawy. Oficyna Wydawnicza Politechniki Warszawskiej. 2019.

13. Tryapkin EY, Keino MY, Protasov FA. Synchronous phase measurements in the automated monitoring system of railway power supply facilities. Russian Electrical Engineering. 2016; 87(2):110-112. https://doi.org/10.3103/S1068371216020176.

14. Pawlik M. Cybersecurity guidelines for the employees of the railway entities. Railway Report. 2021;191:9597. https://doi.org/10.36137/1915E.

15. Bialon A, Kuznetsov V, Sychenko V, Hubskyi P. Energy efficient distributed DC traction power supply system. Transport Means - Proceedings of the International Conference. 2019.

16. Su H, Dong H, Zhao F. Research and practice on curriculum integration on power system analysis and electrified railway power supply system. First International Workshop on Education Technology and Computer Science. 2009.

17. Stasiuk AI, Hryshchuk, RV, Goncharova LL. A Mathematical Cybersecurity Model of a Computer Network for the Control of Power Supply of Traction Substations. New Means Cybernetics, Informatics, Computers Engineering And Systems Analysis. Springer Science. 2017;53(3):476-484. https://doi.org/10.1007/s10559-017-9949-z.

18. Pukhov GE. Taylor transformations and their application in electrical engineering and electronics Naukova dumka. Kiev. 1978. (in Russian).

Received 2021-06-29

Accepted 2021-11-05

Available online 2021-11-29



Mykhailo SOPEL dr.hab.

Small Private Enterprise

"ANIGER" Scientific adviser

His research interests concern:

diagnostic of power supply

systems E-mail: regina@

regina.org.ua



Oleksandr STASYUK prof., dr.hab. Automation and Computer-Integrated

Technologies of Transport department, State University Infrastructure and Technologies Obtained dr.hab. degree in 1987.

His research interests concern: Mathematical Models and Methods of Formation of Intelligent Computer Networks for Control of Power Supply and Optimization of Power Consumption of Railways Email: Stas1946@lanet.kiev.ua

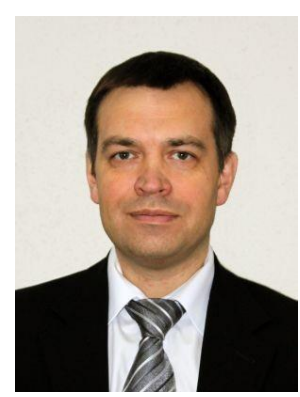

Valeriy KUZNETSOV. prof., dr.hab. Electric Power Department, Railway Research Institute, Poland. Obtained dr.hab. degree in electric transport in 2012. His research interests concern: Energy saving methods, energy management, reliability of traction power supply systems. Email:vkuznetsov@ikolej.pl



Lidiya GONCHAROVA $\mathrm{Ph}$ D,. Automation and ComputerIntegrated Technologies of Transport department, State University Infrastructure and Technologies. Obtained Ph D, degree in "computer systems and components" in 2011. Her research interests concern: Computer monitoring of highspeed technological processes of railway power supplyemail: ktarael@yandex.ru



Petro HUBSKYI. Ph D, Electric Power Department, Railway Research Institute, Poland. Obtained $\mathrm{Ph} \mathrm{D}$,. degree in electric transport in 2019. His research interests concern: Energy saving methods, reliability of traction power supply systems. E-mail: phubskyi@ikolej.pl 